\title{
Space OSPF: An Area Hierarchic Routing Protocol for Routers in Motion
}

\author{
Nouman Bantan and Javed I. Khan \\ Media Communications and Networking Research Laboratory \\ Department of Computer Science \\ Kent State University, Kent, OH 44242 \\ nbantan|javed@kent.edu
}

\begin{abstract}
[Abstract] The research presents a routing protocol for exchange of routing information among vastly spread routers which may also be in continuous motion. We call it Space OSPF. The proposed protocol uses trajectory and supports calculation of approximated optimum path routing tables ahead of time or on-demand withstanding topological changes due to continuous motion. Since the trajectory of space crafts are either predictable (e.g., satellite constellation orbiting a planet), preset (e.g., Space Station), or set on demand (e.g., a space shuttle), the suggested routing protocol enables routers on board those spacecrafts to calculates the time intervals where spacecrafts are in direct view with each other and expected delay. Additionally, this protocol divides the routing domain (e.g., our solar system) into hierarchical routing areas. The hierarchical area provides necessary localization to efficiently and orderly distribute routing information throughout the vast space routing domain. The proposed protocol is suitable for building a space communication infrastructure and provides support for intermittent pathways. The paper presents the principal elements of this protocol along with its stability analysis.
\end{abstract}

\section{Introduction}

T the future, there are plans to launch long-distance exploratory probes, build space colonies on distant planets, and place satellites around planets other than Earth. Like the Mars Lander, these space technologies will gather data and beam it back to Earth by suitable mean. To facilitate these goals, there is a need to construct a routing infrastructure explicitly for a space routing domain that provides efficient routing decisions with low delay and high throughput. An advanced, integrated, communications infrastructure will enable the reliable, multipoint, high data rate capabilities needed on demand to provide continuous, maximum coverage of areas of concentrated activities of in-space outposts on celestial objects (e.g., Mars). ${ }^{1}$

Comparing with Earth-like network, two of the major distinguishing aspects of routing in space are the orbital motion of the routers, and the occlusion which causes intermittent connectivity between routers. On earth, mobileend-points are moving. In contrast in space, routers are moving. Though there are several successful Earth protocols for mobile transport such as Mobile Internet Protocol (MIP) and its numerous variants, there is yet a routing protocol that meets the requirements of the space routing domain, when routers themselves are in motion.

The proposed routing protocol deals with a number of novel challenges that arises in space communication. Some of these are convergence, storage overhead, computational overhead, and transmission overhead ${ }^{2}$. Moreover, energy consumption is an integral part of a satellite which means that minimizing computational and transmission overheads are required. Also, it may be required that a router on board a space craft has to store transit traffic for long periods of time. All these issues have to be addressed by the functionalities supported by the routing protocol. In the context of space communication, any routing schema faces the following five questions: 1) will the routing protocol use network state information? 2) Where is the routing table created/computed? 3) Will the routes have intermittent links? 4) Will the routing protocol support long delays? 5) Will the routing protocol use the predictable aspects of space objects?

The use of network state information can be either static or adaptive. Static routing does not have mechanism to automatically adapt to the dynamic changes inside a network. The network state is made of information about the topology (e.g., which router is connected to which router) and information about the traffic (e.g., how is the traffic load distributed among the network elements). Adaptive routing can be either isolated or non-isolated. Isolated routing are adaptive algorithms that base their routing decisions on local information such as the length of the 
queues in the router's output interfaces (e.g., Hot Potato routing,). Non-isolated algorithms take into account the status of the network at an extent ranging from the neighboring routers to the whole network. Most of today's routing algorithms in terrestrial networks are non-isolated.

Another important issue is the location of the routing table's calculation. These calculations can be centralized, decentralized or distributed. In a centralized approach, the routing tables for all routers are created statically at a central location. In a decentralized approach, each router calculates its routing table statically with no cooperation from others. In a distributed approach, a set of routers cooperate together in order to compute the routing table.

Another important functionality especially for space communication is the support of intermittent pathway. TCP/IP networks have been designed with a specific assumption that all routes in the routing table contain no intermittent paths. In space a pair of satellites who wish to communicate with

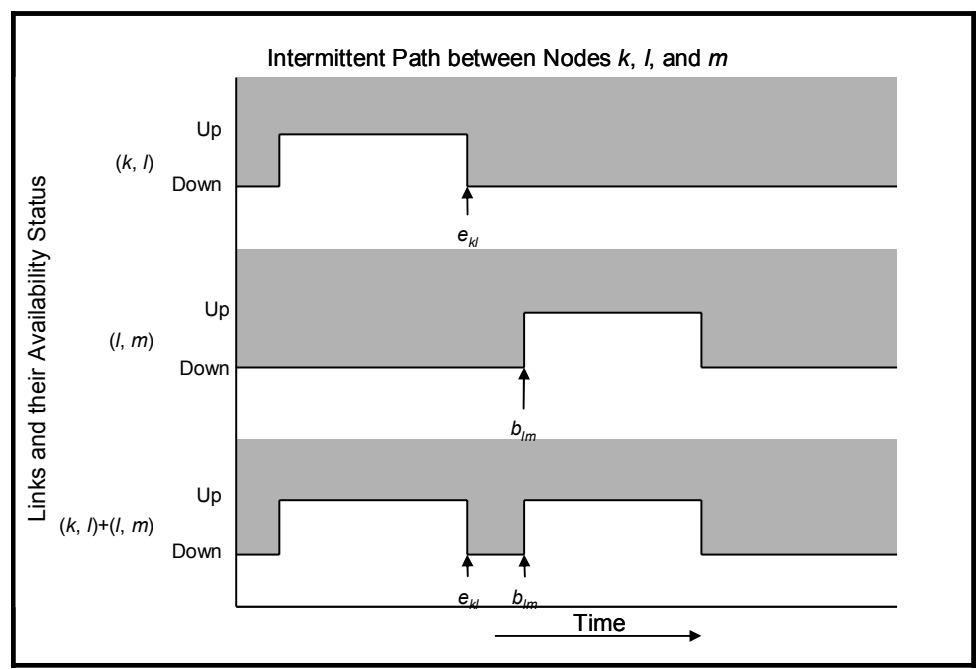

Figure 1: An intermittent path example. each other, may not have direct view of each other which may cause links between them to be intermittent. If a routing protocol provides support for intermittent pathways, it must support long delays. In space, propagation delay between a pair of routers may take hours which will cause many problems, like low throughput, loss of security synchronization, and longer convergence period ${ }^{3}$. In essence, a space routing protocol has to provide a network topology structure or method to deal with long delays.

Another key functionality for space communication is the predictability of space objects. All space objects are moving according to mathematical equations and their positions in space are calculated with extreme precision ${ }^{4}$. It is advantageous to include predictability to a routing protocol because routes can be calculated ahead of time but at the cost of incurring computation overhead. In centralized routing, predicting the location of all routers is overwhelming worrying because of the single point of failure phenomena. Decentralize routing is a better solution but it reiterates the computational overhead at the router where energy limitation on board spacecrafts is an issue ${ }^{5}$. Distributive routing may be better than centralized and decentralized routing because the predictability computation is divided between routers.

\section{A. Related Works in Space Routing}

There are several interesting space routing research which tackles only selected aspects of routing involving constellation satellites orbiting Earth and in deep space. However, it seems only two- ASCoT architecture ${ }^{6}$ and space time routing framework ${ }^{7}$ (STRF) encompass a total solution required towards a routing protocol for space. ASCoT depends on underlying systems which provide a variety of information and services to the ASCoT middleware. The basic idea behind ASCoT is that link trajectories, together with link attributes, are disseminated throughout the network where each router can then independently compute a path to a given location by computing a shortest path tree (using a modified Dijkstra's algorithm) spanning the network. The STRF ${ }^{7}$ leverages the predictability in router motion where it constructs a space-time routing tables where the next hop is selected from the current as well as the possible future neighbors. Although, the ASCoT is the closest in comparison with the proposed research, in the proposed research all the information and services are built within the protocol. Furthermore, the proposed research provides support for intermittent path but ASCoT does not. The predictably model for intermittent connections, which is used in STRF, is similar to the proposed research. However, STRF is based on hop count. In STRF, all hops are assumed to be equal cost the same message size which is troublesome in space environment since delays in a space hop can vary wildly in inter-planetary network. None addresses hierarchical distribution of routing information propagation- which is important in scalable vast domain routing. $\mathrm{OSPF}^{8}$ is the most dominant current earth Internet routing protocol. One of the major advantages it offered over its predecessors (RIP) is area division. However, $\mathrm{OSPF}^{4}$ itself faces non-trivial challenge in supporting intermittent pathway, continuous router motion, and hierarchical area structure 4 .

In this paper we present a new routing protocol, inspired by OSPF's area concept, which consider the complex challenges of space and presents a routing protocol for exchange of routing information among vastly spread router 
community where routers can be in continuous motion- we call it Space OSPF (SOSPF). The proposed protocol uses motion models and supports calculation of approximated optimum path routing tables ahead of time or on-demand withstanding topological changes due to continuous motion. Since the trajectory of space crafts are either predictable (e.g., satellite constellation orbiting a planet), preset (e.g., Space Station), or set on demand (e.g., a space shuttle), the suggested routing protocol enables routers on board those spacecrafts to calculates the time intervals where spacecrafts are in direct view with each other and expected delay. Additionally, this protocol divides the routing domain (e.g., our solar system) into more powerful hierarchical routing areas. The hierarchical area provides necessary localization to efficiently and orderly distribute routing information throughout the vast space routing domain mapping to space's natural hierarchical topological organization. The proposed protocol also provides necessary support for intermittent pathways based route computation. In this paper, section -II describes the key novel aspects of this protocol $^{11}$ including (a) concepts of celestial area hierarchy, (b) the modified hello protocol, (c) motion model based mobility and new link state

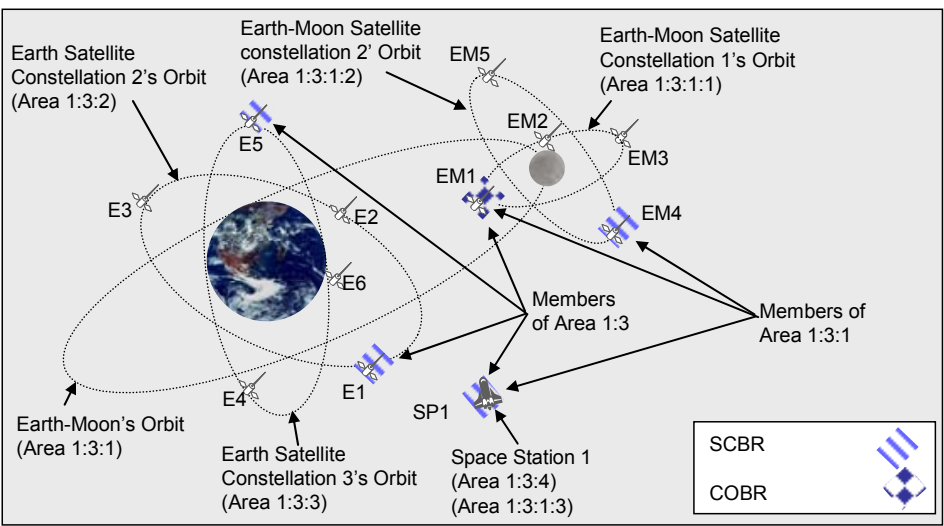

Figure 2: An example of an Earth celestial object area. advertisement (LSA), and (d) support for intermittent pathway routing. Then section-III provides stability analysis of the protocol both analytically as well as experimentally.

\section{The Area Hierarchal Routing Protocol for Routers in Motion}

The solar system encompasses the Sun and the set of celestial objects gravitationally bound to it. Those celestial objects are planets and their moons and thousands of small bodies which consist of asteroids, meteoroids, comets, and interplanetary dust. In the future, there are plans to network the whole solar system and beyond. Consequently, there are a number of studies and suggestions that will make the solar system network-ready which are 1) establishing colonies on planets and moons, 2) positioning satellite around planets other than Earth, 3) positioning space stations in deep space, 4) have space shuttles travel safely into deep space, and 5) positioning satellites in the Asteroid Belt.

In order to for all of these spacecrafts to communicate with each other, we built a routing protocol that provides an infrastructure that is capable of such task.

\section{A. Hierarchical Area Structure}

In order to network the solar system, there must be a network infrastructure which enables such task. Consequently, the solar system is divided into a hierarchical structure by nature. At the time of this research and to our knowledge, there is no space routing protocol that takes advantage of the hierarchical structure of the solar system. The proposed space routing protocol is taking advantage of this hierarchical structure by placing all celestial objects in a hierarchical area structure. Moreover, the suggested routing protocol categorizes objects in space (natural and artificial) into three area classes which are:

- Satellite constellation area is a set of one or more satellites that share a common orbit. This class includes satellites formations, space stations, and space shuttles.

- Colony area is a network that is placed on the surface of a celestial object. It can be earth's Internet. It also can be as small as an on-board network in various spacecrafts. The internal routing inside a colony area can be handled by conventional routing or by SOSPF.

- Celestial object area is a set of representatives of other areas which is formed for a particular celestial object (e.g., Earth, Earth-Moon, or Mars). For a celestial object area $A$ that is formed for a celestial object $C$, the members of $A$ are 1) one or more of the members of satellite constellation area whose members are orbiting $C, 2$ ) members of any satellite constellation area by configuration (e.g., a space shuttle) 3) one or more members of a colony area, which is formed on a colony that is located on the surface of $C, 4$ ) one or more members of celestial objects areas which are gravitationally bound to $C$. 
Table 1: The Earth area example's area memberships

\begin{tabular}{|l|l|}
\hline \multicolumn{1}{|c|}{ Area } & \multicolumn{1}{c|}{ Members } \\
\hline $1: 3$ & $\mathrm{E} 1, \mathrm{E} 5, \mathrm{SP} 1$, and EM1 \\
\hline $1: 3: 1$ & $\mathrm{SP} 1, \mathrm{EM} 1$ and EM4 \\
\hline $1: 3: 2$ & $\mathrm{E} 1, \mathrm{E} 2$, and E3 \\
\hline $1: 3: 3$ & $\mathrm{E} 4, \mathrm{E} 5$, and E6 \\
\hline $1: 3: 4$ & $\mathrm{SP} 1$ \\
\hline $1: 3: 1: 1$ & $\mathrm{EM} 1, \mathrm{EM} 2$, and EM3 \\
\hline $1: 3: 1: 2$ & $\mathrm{EM} 4$ and EM5 \\
\hline $1: 3: 1: 3$ & $\mathrm{SP} 1$ \\
\hline
\end{tabular}

Figure 1 illustrates the hierarchical area structure which can be seen as a recursive structure. In other words, a celestial object area may contain other celestial object areas. In this paper, there is one celestial object area that is placed at the top the area hierarchy (i.e., it has no parent area). This area is referred to as the backbone area. An example of a backbone area is the Sun celestial object area in the solar system.

\section{B. Area Border Routers}

The purpose of areas in the suggested routing protocol is to maintain a level of information hiding and minimize protocol proprietary traffic, as defined in OSPFv $3{ }^{8}$. In turn, protocol traffic within one area does not affect the performance of other areas. Nevertheless, a summary of routing information (e.g., propagation delays between area members and network IPv6 prefixes) must be propagated outside the area. The inherited concept of "area border router" from OSPFv3 is still maintained. In OSPFv3, router $x$, which belongs to area $a$, is configured to be an area border router that summarizes the routing information of area $a$ and propagates it into the backbone. Moreover, when area border routers receive this routing information, they propagate it into the areas which they belong to. At the end within the AS, all routers have identical view of the AS's routing domain.

An area border router is a router on board a spacecraft (referred to as space router hereafter) which belongs to area $A$, summarizes the routing information of $A$, and propagates it to its parent area. Although area border routers for a satellite constellation areas and celestial object areas has the same functionalities, but they differ in their role in the area hierarchy.

\section{Satellite Constellation Border Routers}

Since areas are hierarchically structured, every satellite constellation area has at least one Space router which summarizes the routing information of the area it belongs to and propagates it into it its parent area (a celestial object area). A space router that is configured for this task is called a satellite constellation border router (SCBR). Furthermore, the suggested routing protocol provides the ability for a space router to be hidden from the routing domain outside its area. Thus, an SCBR only propagates routing information of Space routers who have their DoNotAdvertise ${ }^{*}$ bit set off

\section{Celestial Object Border Routers}

If celestial object area $A$ is a child of another celestial object area $P$, at least one member (and space router) of $A$ must be configured to summarize the routing information of $A$ and propagates it into $P$. A space router that is configured for this task is called a celestial object border router (COBR) $)^{\dagger}$.

An example of a celestial object area and satellite constellation areas are shown in Figure 2. Moreover, Figure 3 shows the area hierarchy of the given example and Table 1 shows the area membership the example. Other than area border routers, the suggested allows space routers to belong to multiple areas as seen in Figure 3 where Space

\footnotetext{
${ }^{*}$ DoNotAdvertise is a bit field that allow routers to be seen only by the areas that they are in

${ }^{\dagger}$ Note that an COBR is also a SCBR
} 
Station 1 belongs to areas 1:3:4 and 1:3:1:3. Moreover, a space router that belongs to multiple areas must create the necessary data structure for each area it belongs to.

Looking at Table 1, EM1 is the COBR for area 1:3:1. Furthermore, EM1 belongs to three areas, 1:3:1:1, 1:3:1 and 1:3 which means that EM1 is configured to represent all Space routers in the Earth-Moon celestial object area in the Earth celestial object area.

When a space router belongs to multiple areas, it causes more protocol traffic cost which might deteriorate the overall performance of the network. Careful planning must be employed in area membership configuration. Otherwise, the network will suffer greatly.

\section{Neighbor Data Structure}

For each pair of Space routers that belong to a common area, a neighboring relationship is formed where a "Neighbor Data Structure" is created at each end. The neighbor data structure consists of all the required information to form an adjacency between a pair of Space routers. Each neighbor data structure has the following parameters:

- Neighboring Router's ID: This the IPv6 address of the neighboring router.

- Area's $I D$ : This is identity of the area which encompasses the router and the neighboring router.

- HelloInterval: This is the length of time between hello packets that the router sends to the neighbor.

- Maximum Stability Period: This is the period of time where the neighbor is checked for changes.

- Propagation List: This is a list of propagation delays at different times to the neighboring router. The list consist of multiple entries where each entry contains the following:

- Birth Time: The clock time when the neighboring router becomes in direct view.

- Propagation Period: The time duration of direct view with the neighboring router without interruption

- Propagation Delay: This is the propagation delay (in seconds) during the Propagation Period. This

period reflects all delays incurred from delivering the maximum size packet to the neighbor (e.g., packet processing delay, packet flight time, transmission delay, and QoS delay)

- New LSAs List: This list contains LSA headers of LSAs that are received when the neighboring router is in Sleeping state.

- LSAs Threshold: This is the maximum number of LSAs that can be held in the New LSAs List

- DoNotAdvertise bit: If set, the neighboring router wishes not to be advertised outside this area which means that the LSAs associated with this neighboring router are not propagated outside this area

1. Neighbor States

In addition to the OSPFv3 neighbor states defined in Ref. 41, there is one extra state called Sleeping (see Figure 4). Both neighboring routers are transitioned to the Sleeping state once the direct view between them is occluded. In reality, this transition is based on the configuration of the Neighboring Routers list. Then, the next state depends on one question which is:

How many changes have had happened in the network since the neighboring router has transitioned to Sleeping state?

The states are listed in the order of progressing functionality. For example, the Down state is listed first followed by a list of intermediate states before the final Full state. The Full state is achieved where the router and the neighboring router are in direct view and theirs link state databases (LSDs) are synchronized. In this paper, the reference of a state being lower or greater than another state depends on the progressing functionality between them. Aside from the Sleeping state, the progression of states is the same as in OSPFv $3^{41}$. After a

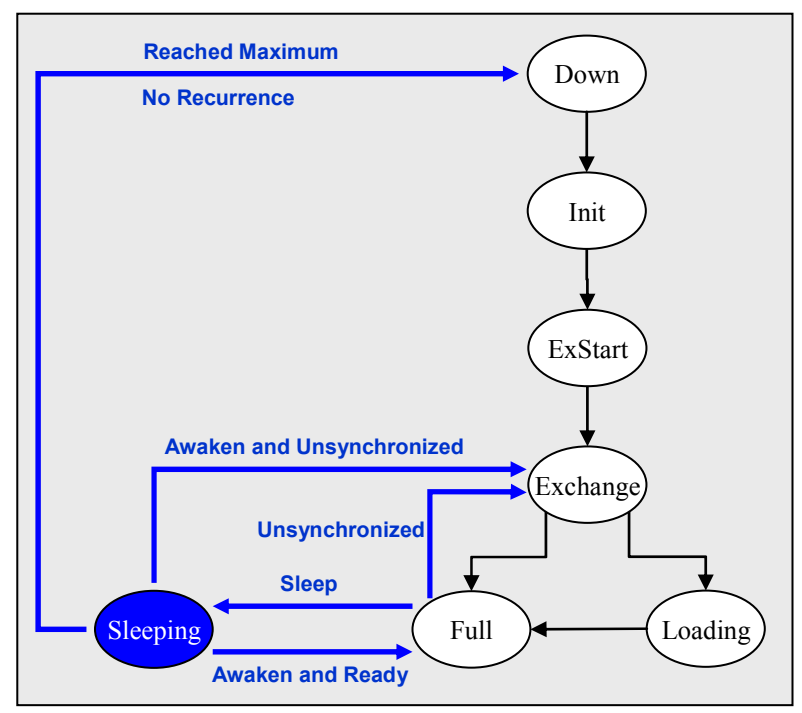

Figure 4: Neighbor State Diagram neighboring router is transitioned from Full to Sleeping, the router may receive LSAs from other Space routers indicating changes in their LSDs (see section G). These LSAs may have to be added to the New LSA list that is

\footnotetext{
${ }^{\ddagger}$ Neighboring Routers list defines which routers must have neighboring relationship with the list’s router.
} 
created for the neighboring router. Furthermore, the neighboring router remains in Sleeping state until one of the following events happen: 1) the number of new/modified LSAs in the New LSA list has been reached LSAs Threshold, 2) the clock time of the next direct view to the neighboring router is reached, and/or 3) the clock time of the next direct view to the neighboring router is unknown

The next neighboring router's state depends on the occurrence of one or more events. At the time of this research, the only LSAs recorded in the New LSA List during Sleeping state are LSAs which are Space Router-LSAs (SR-LSAs) and Area Membership-LSA (AM-LSA) (see section G). Furthermore, only LSAs that are generated by Space routers, and have their DoNotAdvertise bit set on, are inserted in the New LSA List.

\section{Hello Protocol}

For every neighboring relationship between a pair of routers, both of the routers must create and maintain bidirectional relationship. This process is done through the Hello protocol which establishes and maintains neighbor relationships. Maintaining relationships with neighbors is done by exchanging hello packets at regular intervals. It also, ensures that communications between neighbors are bidirectional. A hello packet contains following fields which are: 1) source Space router's IPv6 address, 2) destination Space router's IPv6 address, 3) calculating method tag, 4) DoNotAdvertise bit, 5) area of this bidirectional relationship, 6) source space router's six orbital parameters, and 7) the date and time where the source Space router is at the true angle $\$$

When a space router is turned on, it send hello packet to all members of the Neighboring Routers list. In turn, the receiving router will reply with its own hello packet and bidirectional relationship is established and neighboring relationship is being formed between the pair of routers. Thereafter, hello packets are exchanged to maintain this relationship.

\section{Establishing Bidirectional Relationship}

When Space router $R$ receives a hello packet from Space router $S$, it checks if $S$ 's IPv6 address is in the Neighboring Routers list. If it doesn't exist, then Space router $S$ 's IPv6 address and the six orbital parameters (if $S$ 's calculating method tag is zero) are added to the Neighboring Routers list. Also, if $S$ 's IPv6 address is not in the Space Routers List, then Space router S's router's IPv6 address and its six orbital parameters are added to the Space Routers List.

Furthermore, and $R$ sends a reply hello packet to $S$ and restarts its Hello timer. When $S$ receives $R$ 's hello packets, it restarts its Hello timer. Now, both Space routers $S$ and $R$ have bidirectional relationships with each other.

\section{E. Database Exchange Process}

Once a pair of Space routers has bidirectional relationships, their link state database must be synchronized. Each Space router describes its LSD into a number of network packets, called Database Description packets. Each Database Description packet contains a number of LSAs which were generated by Space routers that wish to be advertised into the Area defined in the hello packet.

Once all the Database Description packets are transmitted, each router transitions the neighbor state of the other router from Exchange state to Loading state. Then each router checks which LSAs need to be updated and request them in Link State Request packets. The recipient of a Link State Request packet finds the requested LSAs in its LSD and transmits them to the neighbor in Link State Update packets. All Link State Update packets must be acknowledged (see section $\mathrm{H}$ ).

During the database exchange, if Space router $x$ 1) receives an AM-LSA for area $i$ that was generated by Space router $y$ and 2) area $i$ 's entry in x's Area Membership list does not contain $y$ in the Area Members field, then $x$ recalculates its Area Membership list and proceed to form bidirectional communication and neighboring relationship with $y$.

If an LSA, other than AM-LSAs, which is generated by a space router that is not in the Space Routers List, the Space router's ID is recorded in New Space routers List. The coordinates of the entries in this list will be requested at the conclusion of the database exchange process. A Coordinate Request packet is sent to the neighbor for every new Space router. Once the Coordinate Request packet is received, a Coordinate Definition packet is sent which contains the calculating method tag and the six orbital parameters of the new Space router (if the calculating method tag is zero). If the calculating method is not zero, the calculating method must be available at the requesting router; otherwise the LSA that caused this packet exchange will be removed from the LSD. Finally at the conclusion of the Database Exchange Process, each router transitions the neighbor state of the other router to Full state.

Moreover, if an SR-LSA is received whilst two neighboring routers are in Full state and the SR-LSA is generated by an space router that is not in the Space Routers List, then the receiving router request the coordinates of

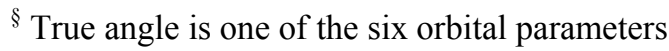


the new router through Coordinate Request packet from its neighboring router which cause each routers to transition the neighbor state of the other router to Loading state. The remaining steps are done as described above.

\section{F. Predictable Motion Induced Mobility}

After all the neighboring relationships are established, each Space router sends its routing information to its neighbors. The routing information depends on the location of the space crafts at different times. Although celestial objects and spacecraft are continuously moving, most of their movement is predictable. All planets, moons, comets, asteroids, and fixed orbit satellites are bound to the six orbital parameters which means that their location can be calculated using those parameters.

Other objects, like space shuttles, space stations, and space probes have trajectories whose locations are determined by a mathematical model or a set of fixed points at different times. In either case, the location of a space object is readily available at any time.

\section{Predictable Model}

The location of a space router must be easily calculated by all Space routers. When a space router introduces itself to another Space router, it uses a hello packet (see section 0) which contains a calculating method tag which corresponds to a calculating model that is used to retrieve the location of this Space router.

The calculation of the location of a space router is not part of the suggested routing protocol, but the calculating model must be available when needed. In this paper, the six orbital parameters are used as the bases of the proposed routing protocol yet those six orbital parameters can be substituted with any other parameters that have the same functionality.

\section{Unpredictable Events}

The unpredictability of the nature of the solar system still exists where events (e.g., galactic cosmic rays and solar wind) can parallelize a satellite indefinitely. In the suggested routing protocol, bidirectional communication between two neighboring Space routers is maintained at all times by exchanging hello packets (see section D). Any disruption at a space router, which has bidirectional relationship with another Space router, is detected when hello packet is not received within a configured waiting period. Once detected, the router will flood the failure to other Space routers. Furthermore, routing tables may have to be recalculated.

\section{G. Advertisements}

The routing information that is sent to all Space routers is sent in the form of an advertisement. These advertisements (called Link State Advertisements (LSAs)) are flooded to a subset of Space routers throughout the routing domain. The suggested routing protocol supports all of the LSAs of the original OSPFv3 which can be found in Ref. 8. This routing protocol addition introduces two new types of LSAs, Space-Router LSA (SR-LSA) and Area-Membership LSA (AM-LSA). The SR-LSA contains the calculation of a space router's links to other Space routers. And, the AM-LSA describes the areas which a space router belongs to.

1. Space Router SR-LSA

An SR-LSA describes the connection between a pair of Space routers where one Space router calculates the clock times of the direct view with another Space router during a Maximum Stability Period. It calculates the estimated propagation delay between them at those clock times. Each SR-LSA contains the IPv6 address of the source router and destination router and number of tuples which describes the link between them at different times. Each tuple consists of three fields which are:

- Begin Time is the clock time when the destination router becomes in direct view with the source router.

- Connection Period is the time period (in seconds) of the connection described in the tuple where the source router and the destination router have direct view of each other. 
- Propagation Delay is the delay incurred from the connection described in this tuple between the source router and the destination router. This delay reflects all delays incurred from delivering the maximum size packet to the destination router which includes packet processing delay, packet flight time, transmission delay, QoS delay, and any other delay that contributes to it.

The number of tuples in an SR-LSA does not exceed MaxTuple ${ }^{* *}$ and the Connection Period does not exceed the Maximum Stability Period ${ }^{\dagger t}$. An example of an SR-

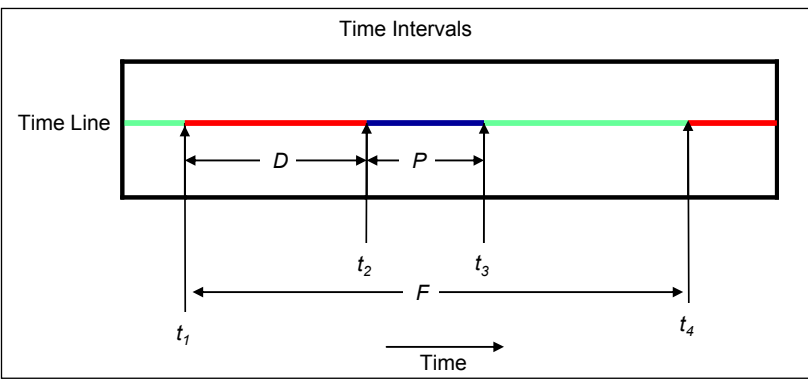

Figure 8: Time Interval layout LSA is shown in Figure 5.

\section{Area-Membership LSA (AM-LSA)}

Each Space router must advertise the areas that it belongs to and for how long. This is done through the Area-Membership LSAs $(\mathrm{AM}-\mathrm{LSA})^{*}$ where each space router advertises the areas that it will part of during the Max Stability Period. The AM-LSA consists of source router's IPv6 address and a number of tuples where each tuple contains four fields:

- Area ID is the area ID of the this tuple

- Area Members contains the list of Space routers' IDs that belong to this area

- Area Start Time is the starting point in time for this area's definition

- Area Period is the time period of the validity of this tuple

AM-LSAs represent the entries of the

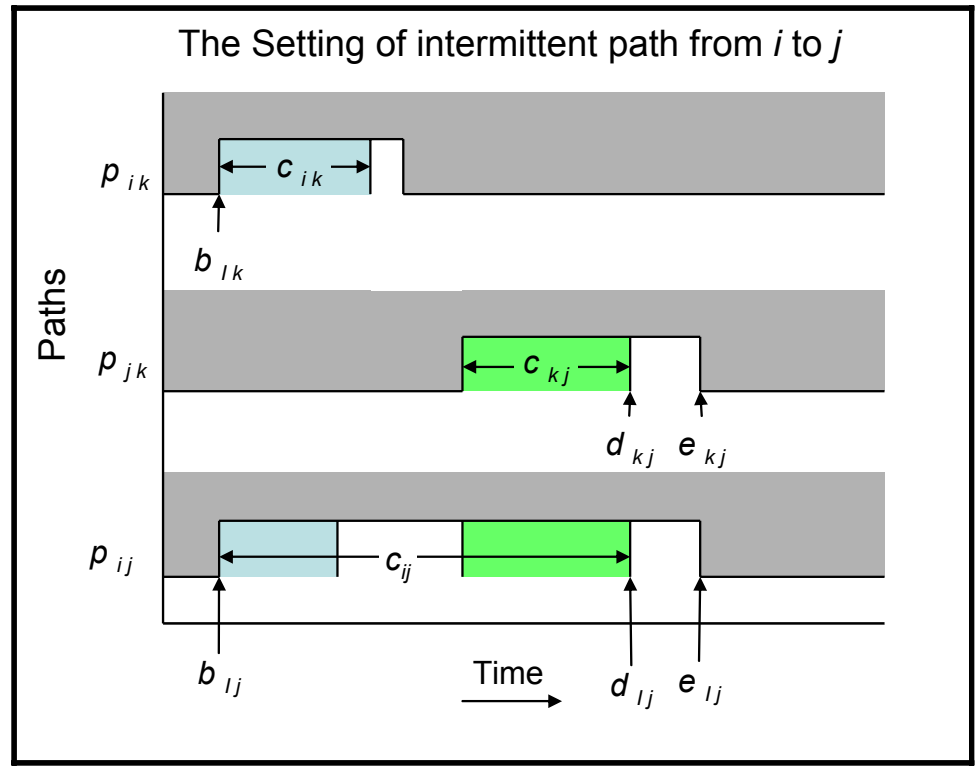

Figure 7: An intermittent path example.

Neighboring Routers list of an area or areas. An example of an AM-LSA for Space router M1 is shown in Figure 6.

Once an LSA is created, it is flooded to the members of the router's area and maybe other areas as well. The flooding procedure is explained next.

\section{H. The Flooding Procedure}

As defined in OSPFv3 ${ }^{8}$, the receiving process of Link State Update packets provides the mechanism for flooding LSAs. A Link State Update packet may contain several distinct LSAs, and may have to flood each LSA one hop further from its point of origination. To make the flooding procedure reliable, each LSA must be acknowledged separately using Link State Acknowledgment packets. Many separate acknowledgments can also be grouped together into a single packet. The flooding procedure LSAs of OSPFv3 are flooded according to the OSPFv3 guidelines ${ }^{8}$.

When an LSA is decided to be flooded, it is flooded according to the area that the router belongs to. There are two types of flooding scopes, internal flooding scope and celestial flooding scope. All Space routers that belong to a satellite constellation area are flooded according to the internal flooding scope. And, All Space routers that belong to a celestial object area are flooded according to the celestial flooding scope.

Internal Flooding Scope: The LSA is forwarded to the Space routers that have bidirectional relationship with (see section 0) except to the Space router at the receiving interface and the LSA's originator. Those routers are defined in the Neighboring Routers list for the specified area.

\footnotetext{
** MaxTuple is a configured parameter which has a default value of $2^{8}-1$

${ }^{\dagger}$ Maximum Stability Period is a configured parameter which has a default value of $2^{18}-1$

\# Not to be confused with Group Membership-LSA
} 
Celestial Flooding Scope: The LSA is forwarded to all Space routers that belong to the router's celestial area (bidirectional relationship is assumed) except to the Space router at the receiving interface and the LSA's originator.

\section{Intermittent Pathway Routing Support}

The suggested routing protocol encompasses a routing algorithm which allows Space routers to use routing information which are received from other Space routers to compute the routing table. This routing algorithm (called Shortest Delay Intermittent Pathway or SDIP) uses the routing information found in SR-LSAs that are received from all Space routers to create routing tables which allows intermittent paths. The SDIP routing algorithm uses a modified version of Floyd-Warshall ${ }^{11}$ shortest path algorithm to create a link between a pair of Space routers. Each link may contain intermediate routers which stores transit data for long periods.

Let the link between two routers $x$ and $y$, be represented by $(x, y)$. Let $b_{x y}$ be the clock time that indicates the beginning of the time period where $(x, y)$ is active. Let $c_{x y}$ be the propagation delay between $x$ and $y$. Let $d_{x y}$ be the clock time (referred to as clock delay) that indicates the estimated arrival time of a transmission between $x$ and $y$. Finally, Let $e_{x y}$ be the clock time that indicates the end of the time period where $(x, y)$ is active. A path is considered intermittent (see Figure 7) if it contains three consecutive routers $i, k$, and $j$, such that:

$$
e_{i k}<b_{k j}
$$

Looking at Figure 7, if the newly found path between $i$ and $j$ has a lower clock delay than the current clock delay, the newly found path becomes the new path between $i$ and $j$.

The SDIP routing algorithm relies on the SR-LSAs which are available in the suggested routing protocol. Looking at Figure 5, all of those parameters which are introduced in this section are deduced from the SR-LSA. Furthermore, the details of the SDIP routing algorithm can be found in Ref. 10.

\section{Analysis}

The main analysis criterion for this research is the stability of the suggested routing protocol. The term Stability can vary by definition from one research to another. For example, in Ref. 9, stability is evaluated by a mixer of 1) network convergence period, 2) routing load on processors, and 3) the number of route flaps. In this paper, stability is defined as follow:

Stability: Stability is the degree to which a system can maintain valid connectivity when disruptions occur.

Primarily, the convergence period is measured between a pair of "network change" events. In the suggested routing protocol, when a link state changes or a link failure is detected, an LSA is generated and encapsulated in a Link State Update packet where it is flooded to the affected areas. Every link update that occurs in the network will require a convergence period before the network enters a stability period.

The convergence period: The convergence period is the amount of time for a router to restore normal traffic handling after recovery from a network interruption.

The stability period: The stability period starts at the time when all Space routers have valid routing table until the next network interruption. Figure 8 illustrates a periodical cycle between convergence periods and stability periods.

Let $t_{1}$ and $t_{4}$ be the clock times of two consecutive network interruptions. Let $F$ be the time interval between the two network interruptions, i.e.,

$$
F=t_{4}-t_{1}
$$

Let $t_{2}$ be the clock time where at least one Space router becomes aware that an interruption happened at $t_{l}$. Let $D$ be the detection time interval for the interruption that happened at $t_{l}$, i.e.

$$
D=t_{2}-t_{1}
$$

Let $t_{3}$ be the clock time where all Space routers have valid routing tables. Let $P$ be the time interval required for all Space router to have valid routing tables from the moment that the interruption was detected, i.e.,

$$
P=t_{3}-t_{2}
$$

Figure 8 shows all parameters that have been defined. Although there are many ways of measuring the stability, in this paper, the measurement of stability is the ratio of time where the network is in a stable period against the period between network interruptions.

Let $\lambda$ be the stability i.e.,

$$
\lambda=\frac{F-(D+P)}{F}
$$

\section{A. Stability Analytical Model}


The model defines a satellite network as a connected directed graph $G=(V, E, A)$, where $V$ is the set of vertices (routers on board satellites), $E$ is the set of edges (direct links), and $A$ is a set areas. Let $n$ be the number of routers in $V$. Let the link connecting router $x$ to router $y$ in $E$ be represented as $(x, y)$. A cost function $c: E \rightarrow R$ is defined, where $R$ is the set of real numbers, determines the cost of $(x, y)$ if $(x, y) \in E$. Let the cost function associated with $(x, y)$ be represented as $c(x, y)$. The cost is a delay metric which is the estimated propagation delay between a pair of routers. An area function $a: V \rightarrow A$ is defined, determine the set of areas of router $x$ if $x \in V$. Let the area function associated with router $x$ be presented as $a(x)$. Let router $x$ and router $y$ belong to a

Table 2: Stability of the network

\begin{tabular}{|c|l|}
\hline Stability Value & \multicolumn{1}{|c|}{ Description } \\
\hline$\lambda=1$ & $\begin{array}{l}\text { The network is free of any updates and there is no } \\
\text { convergence period }\end{array}$ \\
\hline $1>\lambda>0$ & $\begin{array}{l}\text { The network is stable for a period of time. The closer } \lambda \text { is to } 1, \\
\text { the more stable the network }\end{array}$ \\
\hline$\lambda \leq 0$ & $\begin{array}{l}\text { The network is not stable. The updates are occurring before } \\
\text { the previous convergence period is done. }\end{array}$ \\
\hline
\end{tabular}
common area, i.e.,

$$
a(x) \cap a(y) \neq \theta \text { and }(x, y) \in E
$$

Thus, hello packets are exchanged between router $x$ and router $y$ at regular intervals (see section $\mathrm{D}$ ) which is equal to $c(x, y)$. If router $x$ fails, router $y$ will detect the failure when it doesn't receive a hello packet from router $x$ after $k$ multiples of $c(x, y)$. In this paper, it is assumed that $k$ equals 1 . Let $D^{S}$ be the biggest link failure detection interval in the suggested routing protocol where router $x$ and router $y$ are the furthest two closest routers who belong to one common area, i.e.,

$$
D^{S}=\max \left(\min _{y=1, x=1}^{n}(c(x, y)) \text { where } a(x) \cap a(y) \neq \theta\right.
$$

Since Space routers are configured in a hierarchal area structure, not every network interruptions have to be propagated throughout the entire routing domain. If the router $x$ which has its DoNotAdvertise bit set on fails, only the members of its area learn about its failure. Furthermore, let $P^{S}$ the biggest time interval required to have valid routing tables from the moment that router $y$ detected the failure of router $x$ for the suggested routing protocol. Let router $\mathrm{z}$ and router $\mathrm{y}$ be the furthest two routers where router $x$, router $y$, and router $z$ belong to one common area. $P^{S}$ is set to be the summation of propagation delays between the two furthest router in all areas where the failed router $x$ occurred, i.e.,

$$
P^{S}=\sum \max _{y=1, z=1}^{n}(c(y, z)) \text { where } a(y)=a(z)=a(x)
$$

Using Eq. (5), let $\lambda^{S}$ be the stability of the network for the suggested routing protocol, i.e.,

$$
\lambda^{S}=\frac{F-\left(\max \left(\min _{y=1, x=1}^{n}(c(x, y))+\sum \max _{y=1, z=1}^{n}(c(y, z))\right)\right.}{F} \text { where } a(x)=a(y)=a(z)
$$

In the other hand, currently, ground station on Earth send beacon signal periodically to maintain connectivity with satellites, hereafter referred to as referenced technology $(R T)$. Therefore, the link failure detection interval for the referenced technology is the roundtrip propagation delay of a beacon signal. Let $g$ be the Earth ground station and $D^{R T}$ be the biggest link failure detection interval which is the propagation delay between the furthest router $x$ to the Earth ground station $g$, i.e.,

$$
D^{R T}=\max _{x=1}^{n}(c(g, x))
$$

In $R T$, the time interval required to rebuild the routing table is the detection interval which the propagation delay between the furthest router $y$ to the Earth ground station $g$. Let $P^{R T}$ the biggest time interval required to rebuild the routing table for the referenced technology, i.e.,

$$
P^{R T}=\max _{y=1}^{n}(c(g, y))
$$

Also, using formula 38 above, Let $\lambda^{R T}$ be the stability of the network for referenced technology, i.e.,

$$
\lambda^{R T}=\frac{F-2 *\left(\max _{x=1}^{n}(c(g, x))\right)}{F}
$$


As can be seen from Table 2, the closer the stability value gets to 1 the more stable the network is. The stability plays a major role on deciding whether the suggested routing protocol is a feasible solution for space networking. Since there is no statistics about how network interruptions occur between routers on board satellites, there is no valid randomization mechanism which will create a real-life scenario in space.

\section{B. Stability Experiment}

In an abstract view, a satellite network with a diameter of $1013 \mathrm{~km}$ where 5000 Space routers are scattered on the orbits of the eight planets and on the orbit of the Earth-Moon using a uniform random function. Then a failure interval of 6666 seconds is used.

Figure 9 shows the stability of the network after a network interruption which occurred at a space router using stability formula 43 defined in section A. In the worst scenario, Space routers which belong to the backbone area (which means that they belong to areas at four levels) have poor performance. In the contrary, Space routers that belong to area at three levels or less maintain a high stability value even at a network diameter which reaches Neptune.

Furthermore, if a link failure occurred and must be propagated throughout the routing domain, it has the same effect as the worst scenario. Even in the worst scenario, the suggested routing protocol's stability did not go lower than the reference technology's stability.

\section{Conclusion}

This research comprises a space communication protocol which has the three key features 1) defining logical areas, 2) predictable mobility, and 3) distributed computation.

The protocol divides the Solar System which includes celestial objects and spacecrafts into logically separate areas. Within each area, traffic cost is maintained and the routing information of one area can be summarized and propagated outside the area with reduced overhead. Further security is ensured by preventing intruders inside area whose traffic must be secured.

In order to improve the accuracy of the routing tables, the suggested routing protocol incorporates a spacecrafts mobility prediction component that helps extrapolate and pre-calculate routing tables ahead of time, for faster communication.

The proposed routing protocol is the first distributed routing protocol in a space environment. It encompasses dynamic error detection in space and maintains up-to date routing information which includes, but is not limited to changes in spacecraft trajectory, available bandwidth, or any parameter that can affect the spacecrafts routing capability.

The suggested routing protocol requires additional features which are not covered in this paper. One of those features is defining which areas a space router belongs to and for how long. The configuration of these parameters requires placement heuristics which involves time and location in space which is a prime research topic in the near future for the suggested routing protocol to perform efficiently.

Another feature which may require additional support is security. In this research, members of one area can secure a

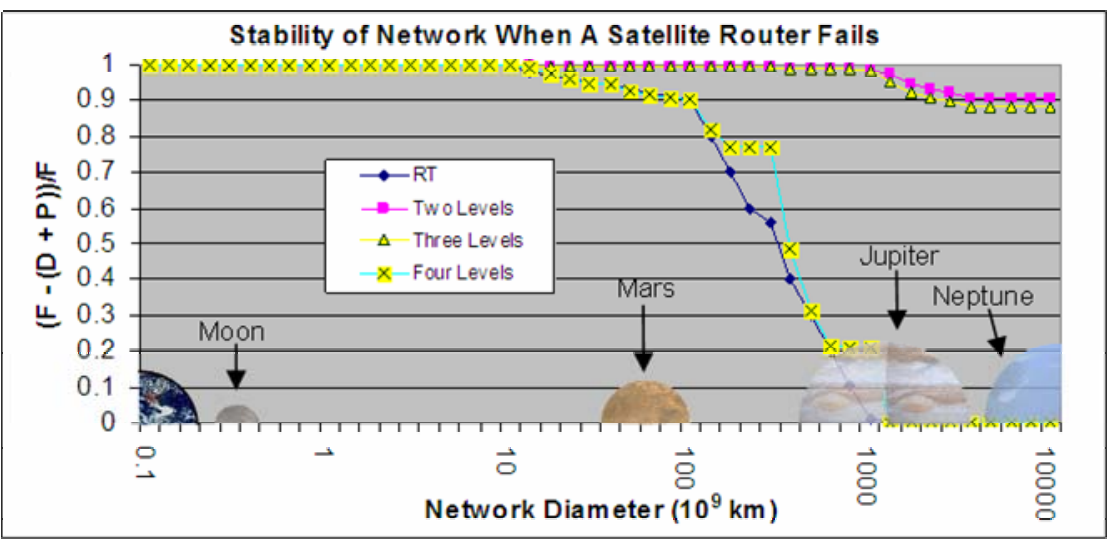

Figure 9: The stability of the network after a failure few of their parameters using AM-LSA which advertises connectivity information prior joining an area. This scenario is the only case where security is thought of in this paper. Although security remains a critical issue in space, security was not a prime concerned at the time of this research. Added security is future research topic which has to be dealt with.

Last but not least, the configuration and definition of space colony was not specified in this paper. In the near future, space colonies and the devices within them have to be defined. Moreover, the interaction between space 
colonies and other spacecraft has to be defined as well. Further research might be implemented along the above directions.

\section{References}

${ }^{1}$ Bhasin, K. and Hayden, J. L., "Developing Architectures and Technologies for an Evolvable NASA Space Communication Infrastructure," Proceedings of The 25th AIAA International Communications Satellite Systems Conference, , Monterey, California, United States, May, 2004.

${ }^{2}$ Krishna, P., Vaidya, N. H., Chatterjee, M., and Pradhan, D. K., "A Cluster-Based Approach For Routing In Dynamic Networks," SIGCOMM Computer Communication Review, Vol. 27, No. 2, April 1997, pp. 49-64.

${ }^{3}$ Sun, Z., Cruickshank, H., Iyengar, S., Howarth, M. P., Claverotte, L., and Gomez, R., "IP Multicast over Satellite," Proceedings of the 21st International Communications Satellite Systems Conference and Exhibit, Yokohama, Japan, 2000, Paper Number: AIAA-2003-2378.

${ }^{4}$ Khan, J., Ellis, B., McFarland L., Putt, C., "A Commentary On The Nature Of Mobility And Connectivity In Space And Implications On The Design Of Space Network Protocols," Technical Report TR2005-08-01, 2005, Kent State University, [available at URL http://medianet.kent.edu/techreports.html].

${ }^{5} \mathrm{Fu}$, A., Modiano, E., and Tsitsiklis, J. N., "Optimal Energy Allocation and Admission Control For Communications Satellites," IEEE/ACM Trans. Netw., Vo. 11, No. 3,June 2003, pp. 488-500.

${ }^{6}$ Gnawali, O., Polyakov, M., Bose, P., and Govindan, R., "Data Centric, Position-Based Routing in Space Networks," Proceedings of the 26th IEEE Aerospace Conference, Big Sky, Montana, United States, 2005, pp. 1322- 1334.

${ }^{7}$ Merugu, S., Ammar, M. H., and Zegura, E. W., "Routing in space and time in networks with predictable mobility," Georgia Institute of Technology Technical Report: GIT-CC-04-07. 2004.

${ }^{8}$ Moy, J. "OSPF Version 2," Internet RFC 2328, 1998, URL: http://www.ietf.org/rfc/rfc2740.txt [cited March 5 2007.]

${ }^{9}$ Basu, A. and Riecke, J. "Stability issues in OSPF routing," Proceedings of the 2001 Conference on Applications, Technologies, Architectures, and Protocols for Computer Communications, San Diego, California, United States, ACM Press, New York, NY, 2001, pp. 225-236.

${ }^{10}$ Floyd, W., "Algorithm 97: shortest path," Communications of the ACM Vol, 5, No. 6, June 1962, pp. 345-370.

${ }^{11}$ Bantan, N., "A Routing Protocol and Routing Algorithm For Space Communication," Ph.D. Dissertation, Department of Computer Science, Kent State University, USA, 2007. 